\title{
Bra.Di.P.O. and P.I.G.R.O.: Innovative Devices for Motor Learning Programs
}

\author{
Guido Belforte, ${ }^{1}$ Gabriella Eula, ${ }^{1}$ Silvia Sirolli, ${ }^{1}$ Paolo Bois, ${ }^{1}$ Elisabetta Geda, ${ }^{2}$ \\ Federico D'Agata, ${ }^{2}$ Franco Cauda, ${ }^{2}$ Sergio Duca, ${ }^{3}$ Marina Zettin, ${ }^{4}$ Roberta Virgilio, ${ }^{4}$ \\ Giuliano Geminiani, ${ }^{2}$ and Katiuscia Sacco ${ }^{2}$ \\ ${ }^{1}$ Department of Mechanical and Aerospace Engineering, Politecnico di Torino, Italy \\ ${ }^{2}$ Department of Psychology, Università di Torino, Italy \\ ${ }^{3}$ Neuroradiology Service, Ospedale Koelliker, Torino, Italy \\ ${ }^{4}$ Puzzle Social Cooperative, Torino, Italy \\ Correspondence should be addressed to Gabriella Eula; gabriella.eula@polito.it
}

Received 14 June 2013; Revised 15 November 2013; Accepted 11 December 2013; Published 11 March 2014

Academic Editor: Ryo Saegusa

Copyright (C) 2014 Guido Belforte et al. This is an open access article distributed under the Creative Commons Attribution License, which permits unrestricted use, distribution, and reproduction in any medium, provided the original work is properly cited.

\begin{abstract}
Two mechatronics prototypes, useful for robotic neurotreatments and new clinical trainings, are here presented. P.I.G.R.O. (pneumatic interactive gait rehabilitation orthosis) is an active exoskeleton with an electropneumatic control. It imposes movements on lower limbs in order to produce in the patient's brain proper motor cortex activation. Bra.Di.P.O. (brain discovery pneumatic orthosis) is an MR-compatible device, designed to improve fMRI (functional magnetic resonance imaging) analysis. The two devices are presented together because both are involved in the study of new robotic treatments of patients affected by ictus or brain stroke or in some motor learning experimental investigations carried out on healthy subjects.
\end{abstract}

\section{Introduction}

According to the theory of neuroplasticity, which neurologists have accepted for only a few decades, the brain is capable of "learning" even in adult age and following injuries, if appropriately stimulated $[1,2]$. In recent years, a number of devices have been developed $[3,4]$ that can stimulate certain functions or simulate physiological movements so locomotor and cognitive functions in brain-damaged patients can be investigated using fMRI, or functional magnetic resonance imaging $[5,6]$.

Functional magnetic resonance imaging has made it possible to look into the human brain "in vivo" for the first time, literally "watching it at work." In addition, fMRI is used on healthy subjects to gain an understanding of our brain's complex capabilities in studies of motor learning.

The paper presents two optimised electropneumatic prototypes, whose previous design can be read in [7-12].

The first one, called Bra.Di.P.O. (brain discovery pneumatic orthosis), is used to move the subject's feet during the
fMRI analysis in order to impose a controlled movement and stimulate the motor cortex during the test.

The other one, called P.I.G.R.O. (pneumatic interactive gait rehabilitation orthosis), is an active electropneumatic exoskeleton for lower limbs motor exercises.

Designed at the Politecnico di Torino, Department of Mechanical and Aerospace Engineering, the two devices have to be used together in the study of motor imagery with healthy subjects or not, to evaluate changes in brain plasticity at the level of motor circuits and motor imagination.

Their common and main advantages are physiotherapist's work improvement; movement imposed with repeatability; electronic data acquisition; continuous measurement of the whole test parameters; different clinical protocols possibility.

In comparison with their previous design [7], these optimised prototypes show the improvements underlined in the following paragraphs.

Furthermore, the clinical procedure here presented, carried out using Bra.Di.P.O. and P.I.G.R.O. on healthy subjects, 
is an interesting research on human motor cortex functioning.

\section{Robotic Machines Prototypes}

2.1. Bra.Di.P.O. Bra.Di.P.O. [7, 9-11] is an MR-compatible device with 1 DoF (degree of freedom) in the sagittal plane, which allows the patient's ankle joint rotation around its proper axis. It has two pedals to which the patient's feet are secured, moved by a pneumatic actuator (Figures 1(a) and $1(\mathrm{~b})$ ), and it operates either in "active mode" (patient follows the pedal movement passively) or in "passive mode" (patient moves the feet autonomously and an optical angular potentiometer, put on the pedals, records the traveled angle).

Some similar examples are shown in literature, as discussed in [13-23].

These devices are always MR compatible, as they have to work in the magnetic resonance chamber without producing interference [13-15] and use in general pneumatic or hydraulic controls.

Bra.Di.P.O. can be adapted to patient's feet with an anthropometric range between 10\%ile woman and 95\%ile man, in agreement with the standards underlined in [24, 25]. All electrical parts and the PC are remotely located. A userfriendly graphical interface is also provided.

A flow chart of the Bra.Di.P.O. management software is shown in Figure 1(c). It was developed by the authors in order to optimize this investigation. Each test starts with a general system check, after which the operator can choose test parameters (cylinder velocity, pedal angle range, and operating frequency). Each frame has a virtual emergency switch to stop software during analysis.

The main problems encountered with the first Bra.Di.P.O. prototype (Figure 1(b)) are referred to: the pneumatic actuator position improvement; the device position adjustment on the scanning table; the dynamics signal transmission outsideinside from the magnetic resonance chamber.

Consequently, a second prototype was designed, as also discussed in [10]. In this second solution, the pneumatic actuator (1) is located inside the device (Figure 2(a)) and it is connected to a bar (2), which rotates around hinge $\mathrm{O}(3)$ to move the pedals (4).

As it was found during the initial fMRI analysis with Bra.Di.P.O., the patient's foot movement could interfere with the test by producing involuntary head movements.

So the device was raised as illustrated in Figure 2(b), because this configuration reduces the transmission of movement from patient's feet to head and solves the problem.

As the raising of the patient's legs also requires a horizontal adjustment of the patient's position on the scanning table (Figure 2(c)), another pneumatic actuator was added under the box and used as motor of a horizontal positioning system.

The final second Bra.Di.P.O. prototype is shown in Figure 3.

Moreover, the problem of a proper dynamics signal transmission through the long tubes here used (about 10 meter), widely presented in literature [16-23] for other similar devices and by authors in [11], was studied and already solved in the first Bra.Di.P.O. prototype.
Here the problem was addressed by selecting nonmagnetic commercial valves and placing them close to the cylinder (PF and PD in Figure 4(a)).

In particular this study was carried out using commercial software, where the layout of the circuit shown in Figure 4(b) was designed.

In this way authors investigated the dynamics performances of Bra.Di.P.O. control circuit, in order to obtain for the patient's feet movement the required working frequency (about $1 \mathrm{~Hz}$ ). It is imposed by paradigms connected to the human motor cortex activation.

In this numerical model, a number of parameters were changed, in order to analyze the system's dynamic behavior varying pedal loads $(m)$, working frequencies $(f)$, supply pressures $\left(p_{s}\right)$, and duty cycles $(d)$. The latter parameter is very important because it directly affects the line supply/discharge time.

During simulation, parameters were varied in the following ranges: mass $m=1-2 \mathrm{~kg}$, working frequency $f=0.2-$ $0.7 \mathrm{~Hz}$, duty cycle $d=0.15 ; 0.25 ; 0.40 ; 0.50$, and relative supply pressure $p_{s}=3.0 * 10^{5} ; 3.5 * 10^{5} ; 4.0 * 10^{5} \mathrm{~Pa}$.

In order to validate the numerical model elaborated, an experimental test bench was constructed and some tests were carried out to compare experimental and theoretical results [11].

An example of results obtained from this study is shown in Figure 5, with $2 \mathrm{~kg}$ load, $3.5 * 10^{5} \mathrm{~Pa}$ supply pressure, $0.5 \mathrm{~Hz}$ frequency, and 0.25 duty cycle [11].

These graphs illustrate a good correspondence between theoretical and experimental results carried out in the same test condition. In fact, varying the various parameters, the shape and the amplitude of the signals are always good and show a proper functioning of the numerical model elaborated by the authors, as can be widely checked in [11].

In particular, analysing Figure 5, Pc (see also Figure 4(a)) reaches the same maximum amplitude value both in the theoretical and experimental tests; Puf (Figure 4(a)) shows a theoretical amplitude value lower than the experimental one; Pud (Figure 4(a)) shows the same behaviour in both cases.

This fact is certainly due to the little errors that sometimes occur between numerical models and experimental tests. The main causes here considered are cylinder and valves friction modeling and physical modeling of a long pneumatic line. Overall results obtained are good and give possibility to construct a final prototype and circuit working always properly.

2.2. P.I.G.R.O. The other prototype here involved is called P.I.G.R.O. and it is an active exoskeleton for lower limbs with an electropneumatic control.

The literature on active lower-limb exoskeletons used for gait rehabilitation, as discussed in [26-31], chiefly deals with machines tethered to a fixed station. They are generally rather rigid, heavy devices and are mostly employed for treadmill training. These systems almost never provide ankle actuation.

By contrast, P.I.G.R.O. system is an innovative device designed for the rehabilitation of patients with clinical problems such as hemiplegia, tetraparesis, and hemiparesis, as well 


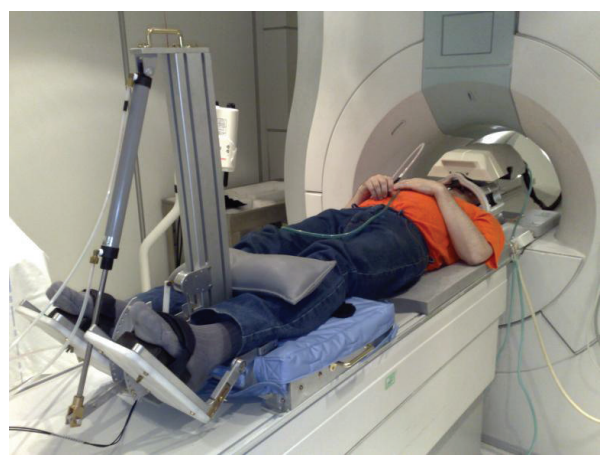

(a)

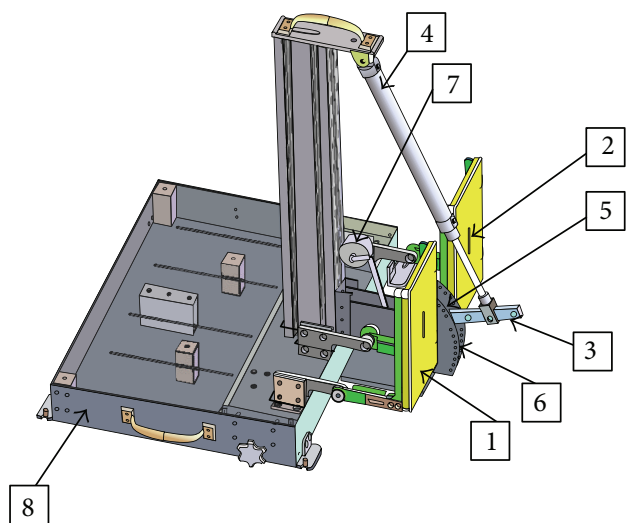

(b)

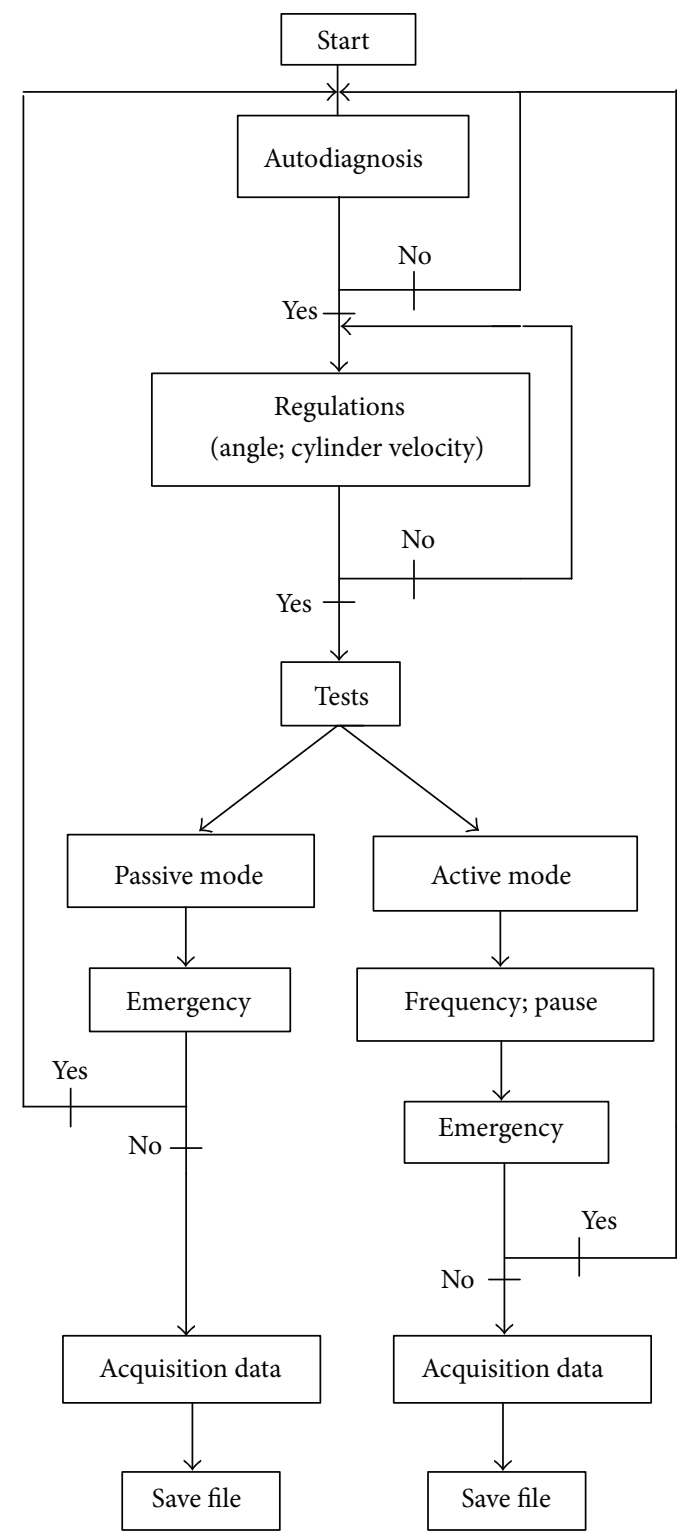

(c)

Figure 1: (a) Bra.Di.P.O. in the magnetic resonance chamber during test. (b) Bra.Di.P.O. first prototype. (c) Bra.Di.P.O. flow chart. 


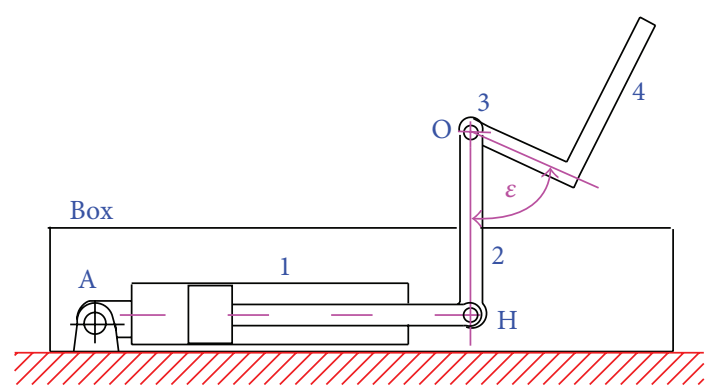

(a)

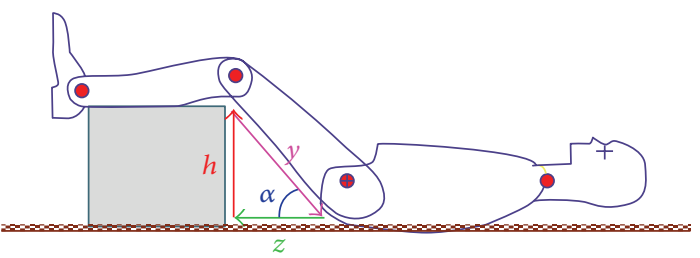

(b)

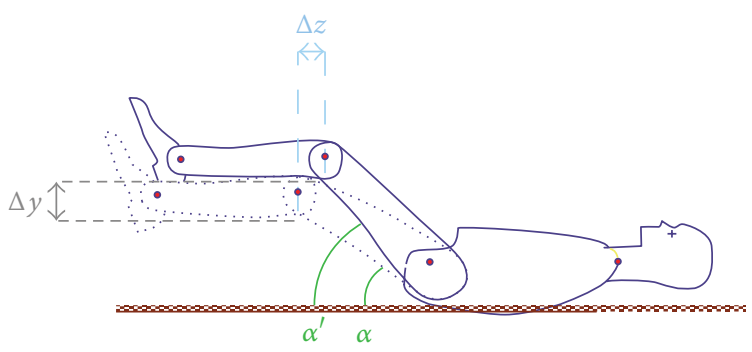

(c)

FIGURE 2: (a) Schematic view of the mechanism underlying the second prototype. (b) Approach used to determine Bra.Di.P.O. vertical position regulation. (c) Horizontal movement of the patient's lower limbs as Bra.Di.P.O. is raised.

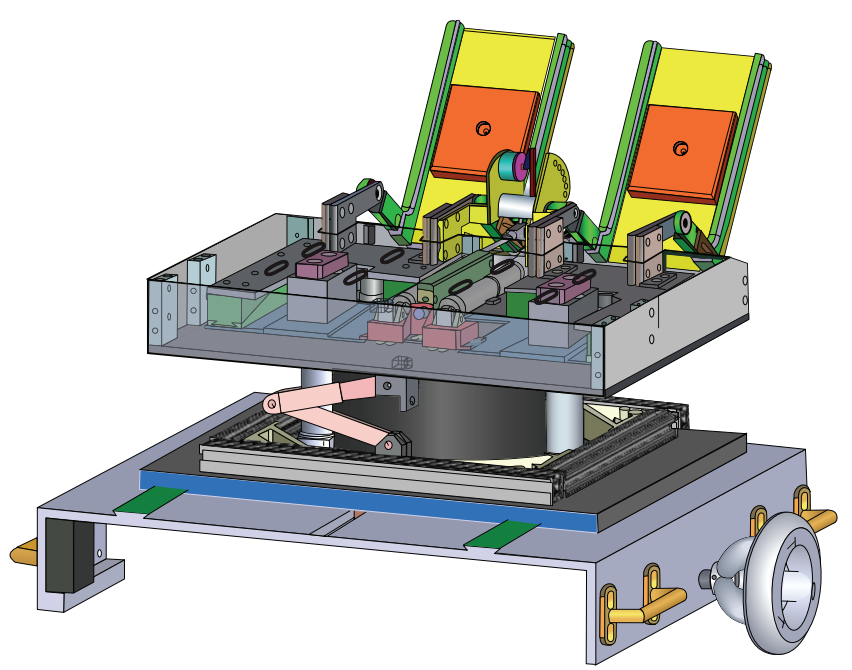

Figure 3: Second Bra.Di.P.O. prototype.

as those who have suffered strokes, ischemias, or cerebral hemorrhages. It can also be extended to certain cases of muscular dystrophy and degenerative motor system disease.

Figure 6 shows some details of the system.

In particular, the system (Figure 6(a)) consists of an electropneumatically controlled active orthosis, a PC to acquire and process data and enable the operator to manage the session, a monitor for the operator, and a monitor for patient biofeedback.

In Figure 6(b) an example of screenshot from the operator's monitor during the test is shown. It is possible to notice the six joints scopes.

In Figures 6(c), 6(d), and 6(e) examples of screenshots from the biofeedback monitor are shown for the patient's
TABLE 1: P.I.G.R.O. adjustments.

\begin{tabular}{lccc}
\hline & $\begin{array}{c}10 \% \text { ile } \\
\text { woman }(\mathrm{mm})\end{array}$ & $\begin{array}{c}\text { 95\%ile man } \\
(\mathrm{mm})\end{array}$ & $\begin{array}{c}\text { Regulation } \\
\text { range }(\mathrm{mm})\end{array}$ \\
\hline Pelvis width & 300 & 650 & 350 \\
Femur length & 330 & 500 & 170 \\
Tibia length & 330 & 500 & 170 \\
\hline
\end{tabular}

hip-knee-ankle. In particular the thicker curve is the machine reference for the patient, while the thinner curve is the patient's performance during the test. In the biofeedback monitor clinicians require a thicker reference curve as range within the patient's performance can be checked during the treatment.

The orthosis is a modular 6-DoF exoskeleton which can be adapted to patients with anthropometric range between $10 \%$ ile woman and $95 \%$ ile man $[24,25]$, as shown in Table 1. All DoF are in the sagittal plane, one for each lower limb joint.

Part of the orthosis structure uses spring steel so that the system can be more readily comfortable and wearable, as well as to allow a certain pelvic movement out of the sagittal plane during the gait cycle.

Depending on therapeutic requirements, P.I.G.R.O. can be used either with the patient suspended or with partial ground contact using a body weight support. In both cases, the body weight support is used to unload the mass of the orthosis as well as that of the patient's body.

As in both types of training the ankle joint actuation is essential for the patient's motor cortex activation; this represents an original and important characteristic of P.I.G.R.O. [32]. 


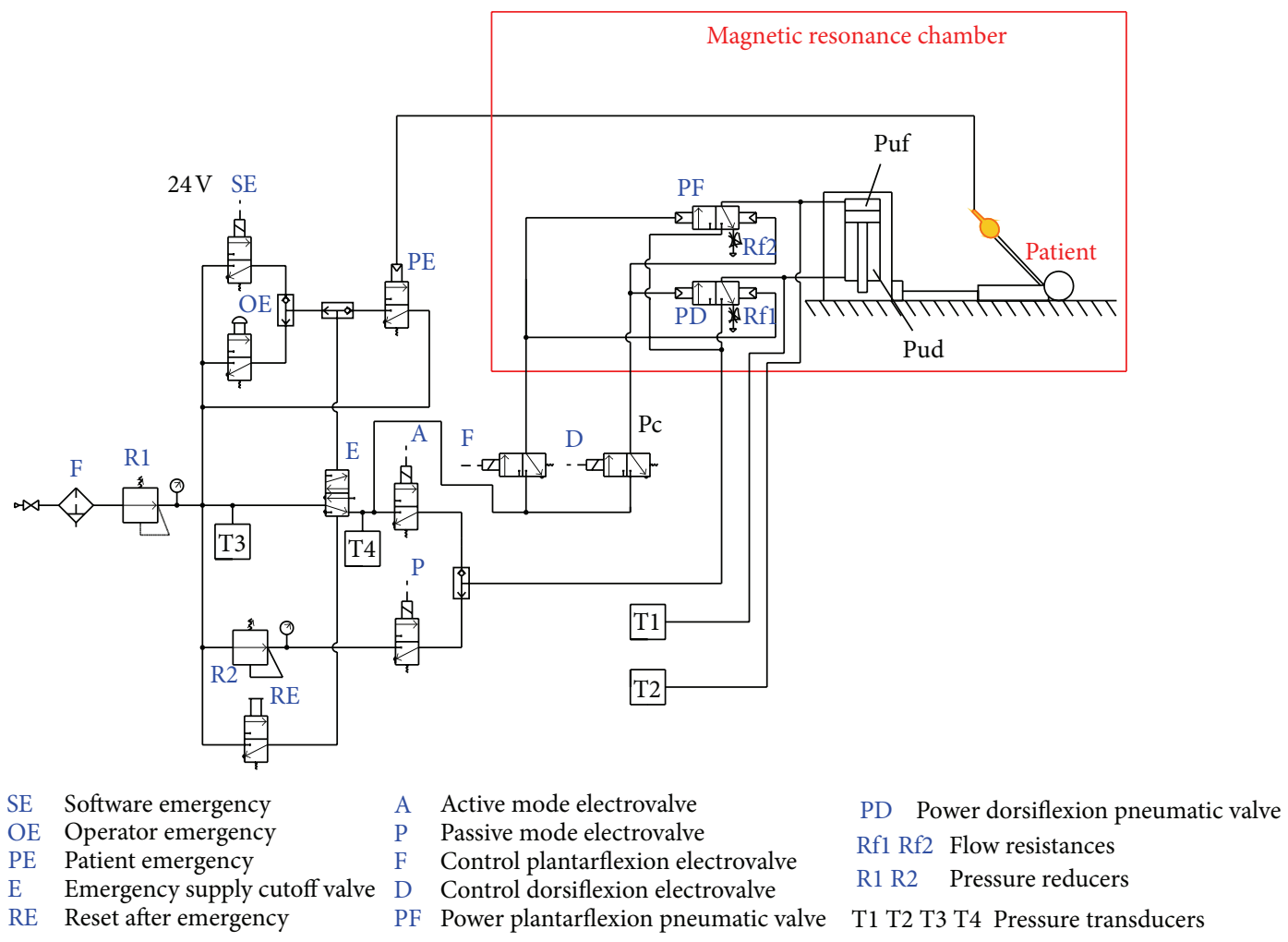

(a)

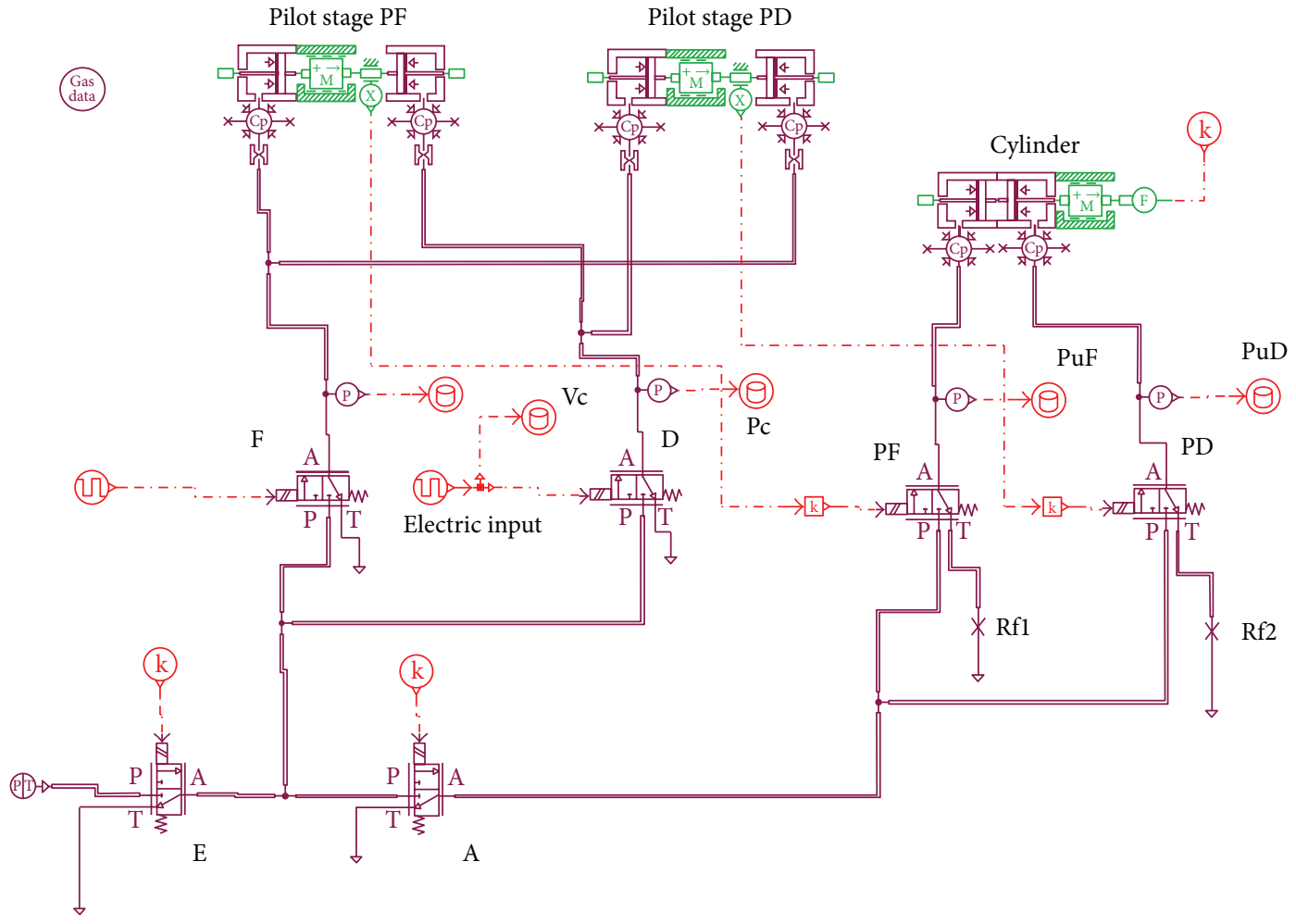

(b)

FIGURE 4: (a) Final electropneumatic control circuit. (b) Layout of the numerical model simulating the optimized circuit. 


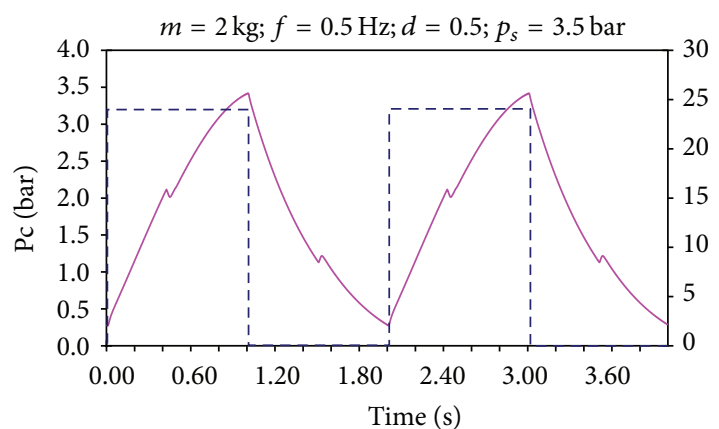

- Pressure Pc - - Electric input

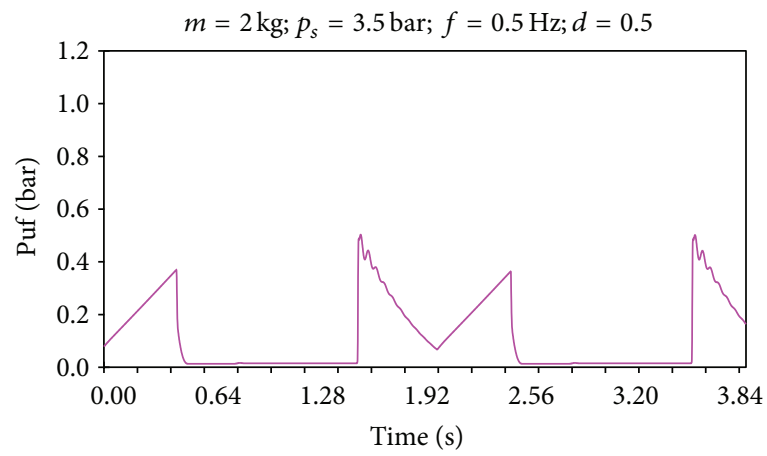

_ Theoretical pressure Puf

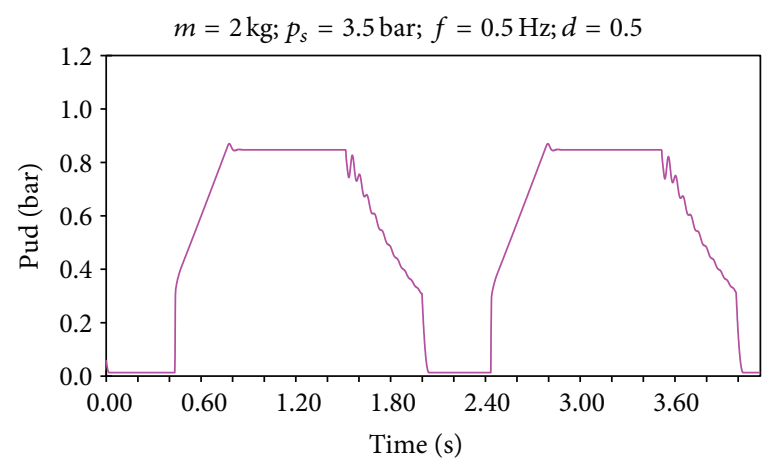

— Theoretical pressure Pud

(a)
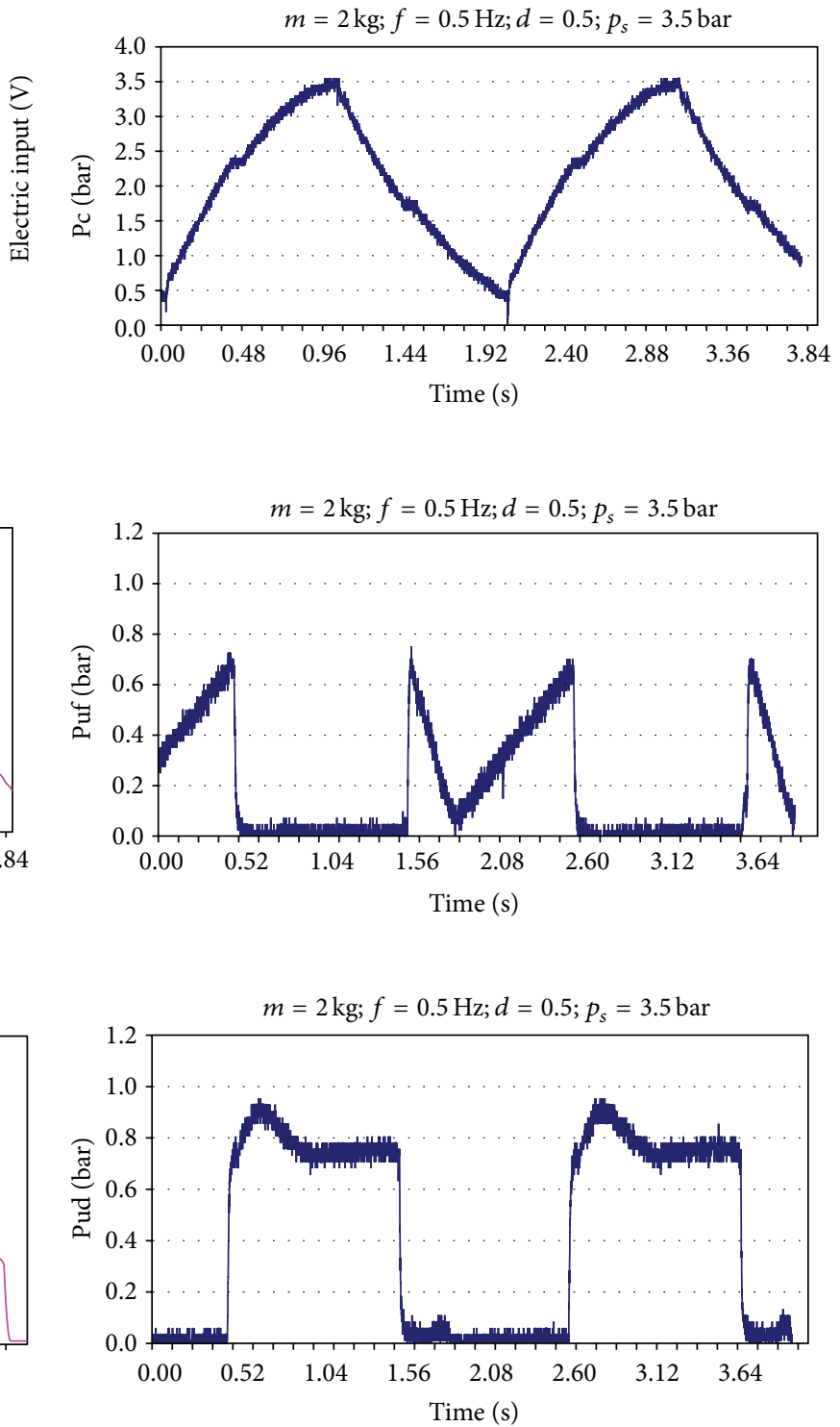

(b)

Figure 5: Theoretical (left) and experimental (right) results with $2 \mathrm{~kg}$ load on actuator, 3.5 bar supply pressure, $0.5 \mathrm{~Hz}$ frequency, and 0.25 duty cycle $\left(1 \mathrm{bar}=10^{5} \mathrm{~Pa}\right)$.

In particular, the ankle joint actuation can also be removed, leaving the patient free to move the foot independently during the treatment, if required.

In particular, overground walking was preferred to walking on treadmill as it allows the patient's advancing in the room and the space provides important sensations and perceptions fundamental to rehabilitation.

The pneumatic actuators operate on the principle of agonist/antagonist muscle pair, thus reducing weight and bulk. The current cylinders could also be replaced with pneumatic muscles if required.

Each leg of the orthosis is equipped with cylinder chamber pressure sensors and position sensors, which track joint movements for use as feedback in system control.
Supply pressure level can be regulated both to vary the force imposed on the patient's legs and to help the physicians' analysis of the patient's autonomous walking progress.

Actuation is electropneumatic but can also be implemented with electric or hydraulic actuators.

The management software is a real-time control where input curves can be either the physiological joints behavior of a standard gait cycle [33] or some other curves choice by clinicians for the training.

Acquired data are sent to the PC via a 10-meter long coded signal transmission cable, multipolar cables, or wireless connections. The software was developed by the authors specifically for this application. It also features an excellent graphical interface to facilitate the operator's work. 

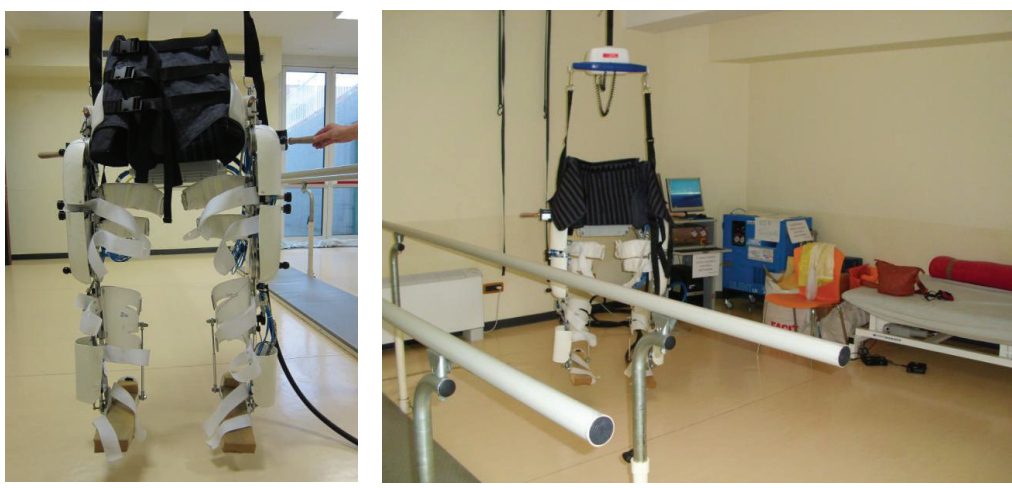

(a)

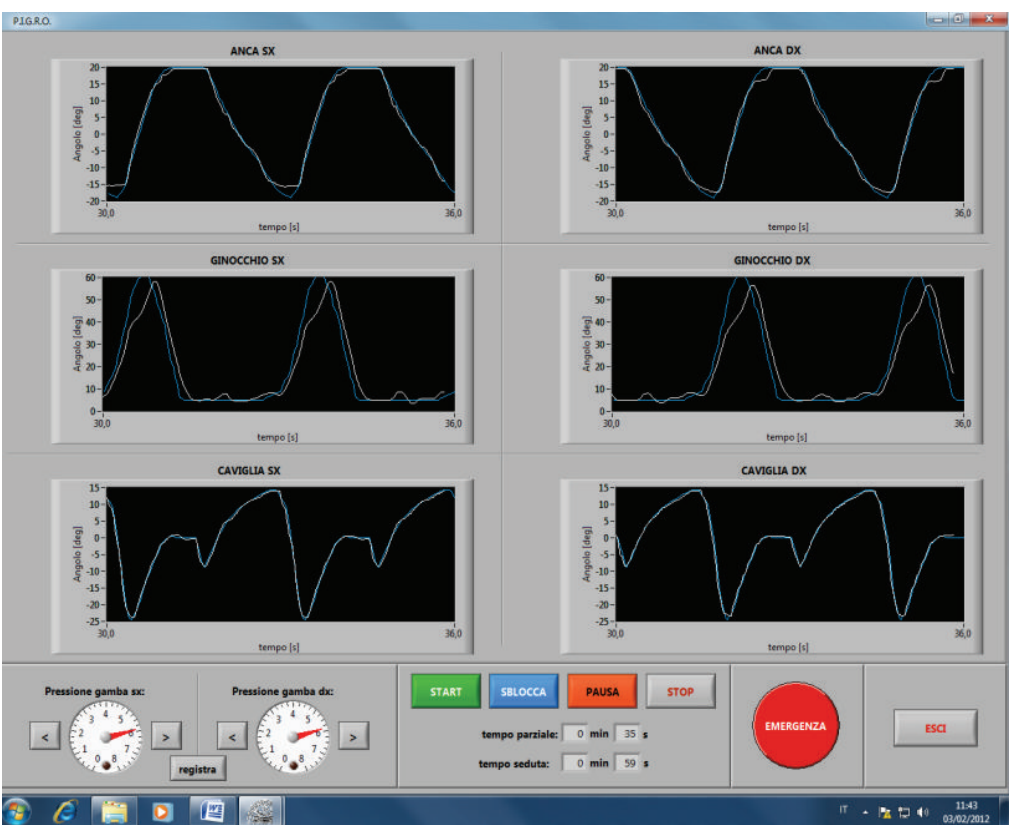

(b)

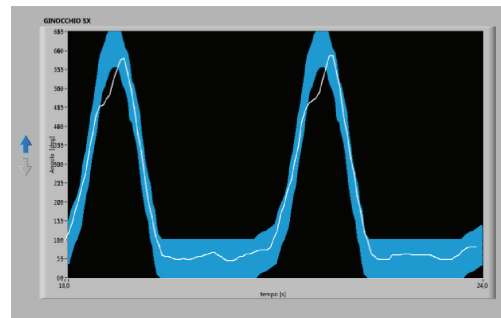

(d)

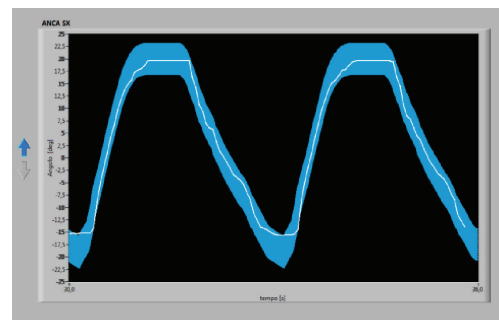

(c)

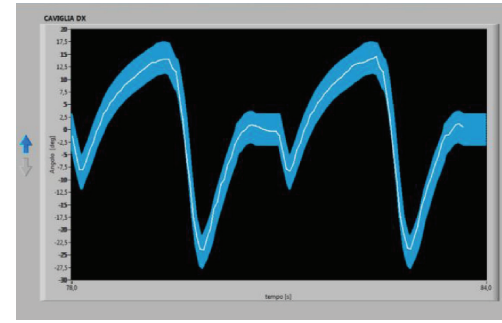

(e)

Figure 6: (a) P.I.G.R.O. in a rehabilitation center. (b) P.I.G.R.O. operator's monitor screenshot with the six joints visualized. ((c),(d), and (e)) Examples of biofeedback monitor screenshots for hip (c), knee (d), and ankle (e).

This graphical interface allows inserting all patient's parameters, especially the anthropometric length of the leg and the patient's weight. In particular patient's mass can influence P.I.G.R.O. movement, both as inertial effects and human interaction with the exoskeleton. So this value is fundamental for the control system.

P.I.G.R.O. does not exceed $30 \mathrm{~kg}$ in weight and it is flexible, versatile, and easy to use.
In particular it must be underlined that this robot is not suitable for the patient's assistance during the day and outside the hospital, as other auxiliary devices on the market.

In fact P.I.G.R.O. is a robotic machine designed for neurorehabilitation training carried out in hospital structures and by clinicians only [32].

Moreover, pneumatic actuation is intrinsically safe and clean, gives a comfortable and soft imposed movement, and 


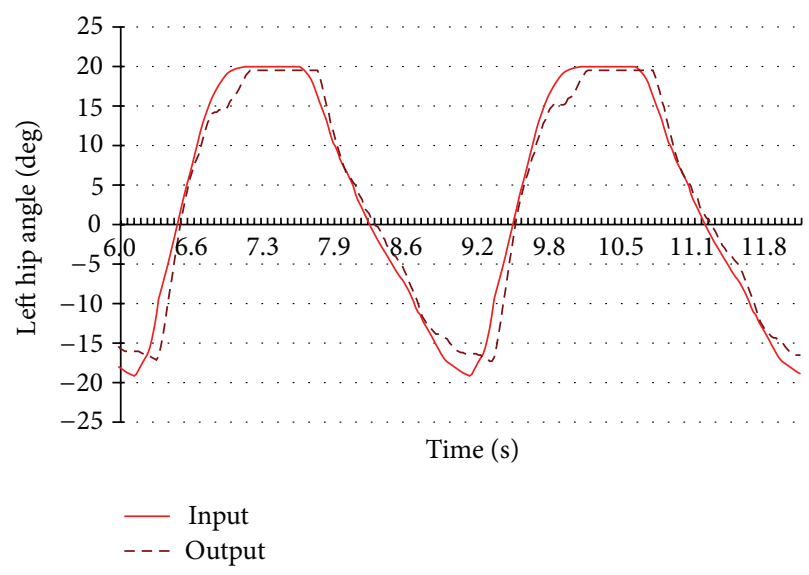

(a)

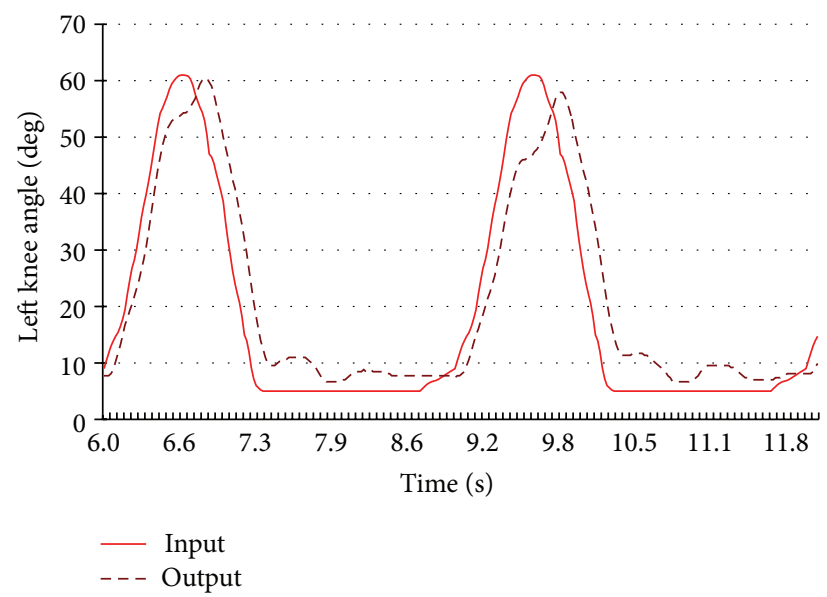

(b)

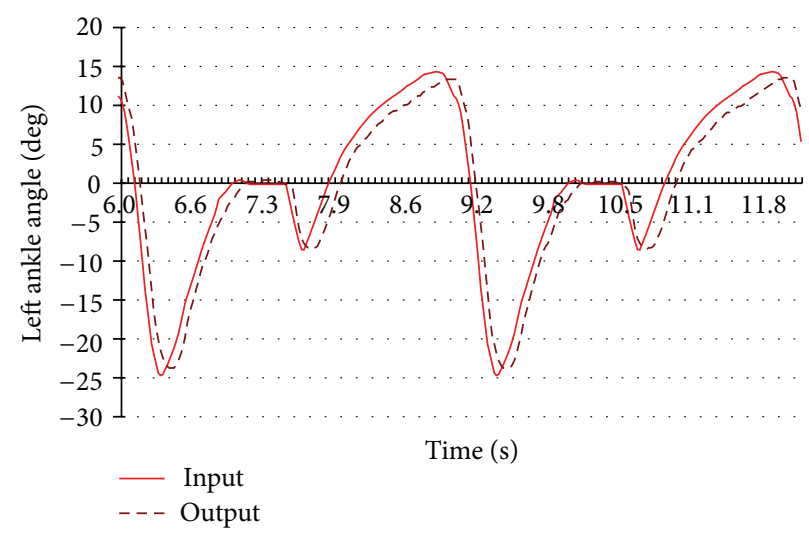

(c)

FIGURE 7: Comparison between P.I.G.R.O. input-output joints angles curves in hip (a), knee (b), and ankle (c).

allows changing forces on patient's legs operating on the supply pressure level.

Figures 7(a), 7(b), and 7(c) show P.I.G.R.O. behavior comparing, during an experimental test, input and output curves for each joint angle. This test was carried out on a healthy subject with a weight of about $70 \mathrm{~kg}$. In particular the graphs referred to a gait cycle of $3 \mathrm{~s}$, analyzed after the initial transient state and to the left patient's leg for simplicity.

These results underline the proper functioning of the system, as the amplitude and the shape of P.I.G.R.O. input curves (control position) and output curves (feedback of the system) are always in full agreement. In particular the small delay between input and output curves is due to the few interactions that anyway occur between a passive conscious subject and a robotic imposed movement.

Finally, the main innovations of this new prototype [32] in comparison with the previous ones $[7,8,12]$ are here underlined.

An important improvement of the human-machine interface design was carried out by authors, studying and testing innovative, ergonomic, and comfortable textiles structures.

A new electric solution, with its own separate emergency switch, was realized for the pelvic adjustment, improving robot wearability and safety.
The control system emergencies were finally defined in three modes: from software; with a pneumatic button; with a patient's button.

An innovative real-time control system was designed, in order to substitute the previous one, based on two PC (master and slave).

P.I.G.R.O. management software was fully reviewed and improved.

In particular, the software is now capable: to slowly start the gait cycle, in order to avoid an initial strong and sudden movement imposed on the patient's legs; to discharge all electro-pneumatic valves, if some emergency situations occur or during the pause required in the training; to stop pneumatic actuators in pressure, when clinicians check the patient's cognitive status.

Furthermore, supply pressure, for one leg or both, can be changed by the operator during the treatment, automatically saving, in a proper patient's memo block, the time of this action and the new pressure level.

A graphical user-friendly interface was designed, in full agreement with clinicians' requirements.

2.3. Comparison of the Two Devices. The main characteristics of the two devices here presented are summarized in Table 2. 
TABLE 2: Technical features of the two prototypes.

\begin{tabular}{ll}
\hline Bra.Di.P.O. & P.I.G.R.O. \\
\hline Nonmagnetic materials and controls & Versatile hardware and software \\
Versatile hardware and software & Good wearability \\
Minimal invasiveness & Minimal invasiveness \\
Easily used emergency controls for operator and patient & Applicable to many clinical situations \\
Remotely located electrical parts & Low weight \\
Adjustable to patient body measurements & Can also be used in the water \\
User-friendly graphical interface & Can be used for both suspended and overground walking \\
Feet can be moved singly or simultaneously in active or passive mode & User-friendly graphical interface \\
Good wearability & Easily used emergency controls for operator and patient \\
\hline
\end{tabular}

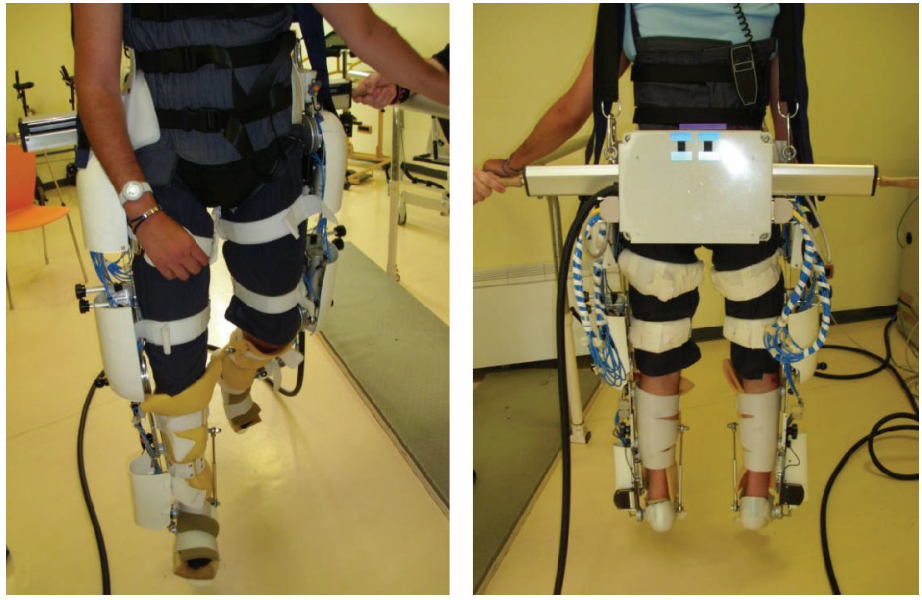

FIGURE 8: Motor training using P.I.G.R.O.

Both Bra.Di.P.O. and P.I.G.R.O. have a good wearability, proper and original management software, and a useful graphical user-friendly interface.

They allow repeating exactly each treatment, to save data, to help and improve the physiotherapist's work.

They are versatile and allow testing and establishing innovative procedure useful for the neurorehabilitation and for the human brain motor cortex study.

The clinical application of these two devices consists of combination of locomotor and cognitive training here preliminarily carried out with healthy subjects, using fMRI to measure changes in plasticity both at the level of the motor cortex and in motor imagery tasks $[34,35]$.

\section{Material and Methods}

3.1. Subjects. Five healthy volunteers (Figure 8) (3 women and 2 men; age range $=20-23$, mean age $=22$ years) took part in the experiment. All subjects were tested and were found to have a sufficient ability to form visual and motor images. Exclusion criteria included history of neurological or developmental illness, mental disorders, drug or alcohol abuse, and current use of medications known to alter neurological activity. All subjects gave informed written consent. The fMRI study was performed at the Koelliker Hospital in Torino (Italy).
3.2. Training. Subjects performed the training tasks using a robotic device (P.I.G.R.O.; see below for a description). The training session consisted of two runs. Each run included active and passive phases. During passive phases, subjects kept their eyes closed and were asked to accommodate movements imposed by the robotic device and to focus on kinesthetic perception. Movements consisted of a sequence of ankle dorsi- and plantarflexions; movements differed in rhythm and speed for the two feet. In the active phase, pressure in the device decreased and subjects had to reproduce the movements learned in the focusing phase, with the same amplitude and speed. Each phase lasted five minutes. The findings from the motor imagery tasks will be discussed below.

3.3. fMRI Assessment. fMRI assessment made use of a motor and motor imagery task: subjects were required to perform ankle dorsiflexion and plantarflexion. In the second session, subjects were required to imagine the same movement. Complete dorsiflexion/plantarflexion cycles should occur with a frequency of about $0.5 \mathrm{~Hz}$. The task was performed using Bra.Di.P.O. Paradigms were performed using a block design with $12 \mathrm{~s}$ of rest alternating with $12 \mathrm{~s}$ of the active condition. Each paradigm consisted of a total of 25 blocks (13 rest conditions, 12 active conditions); 4 functional volumes were scanned during each block; each paradigm lasted 5 min. 

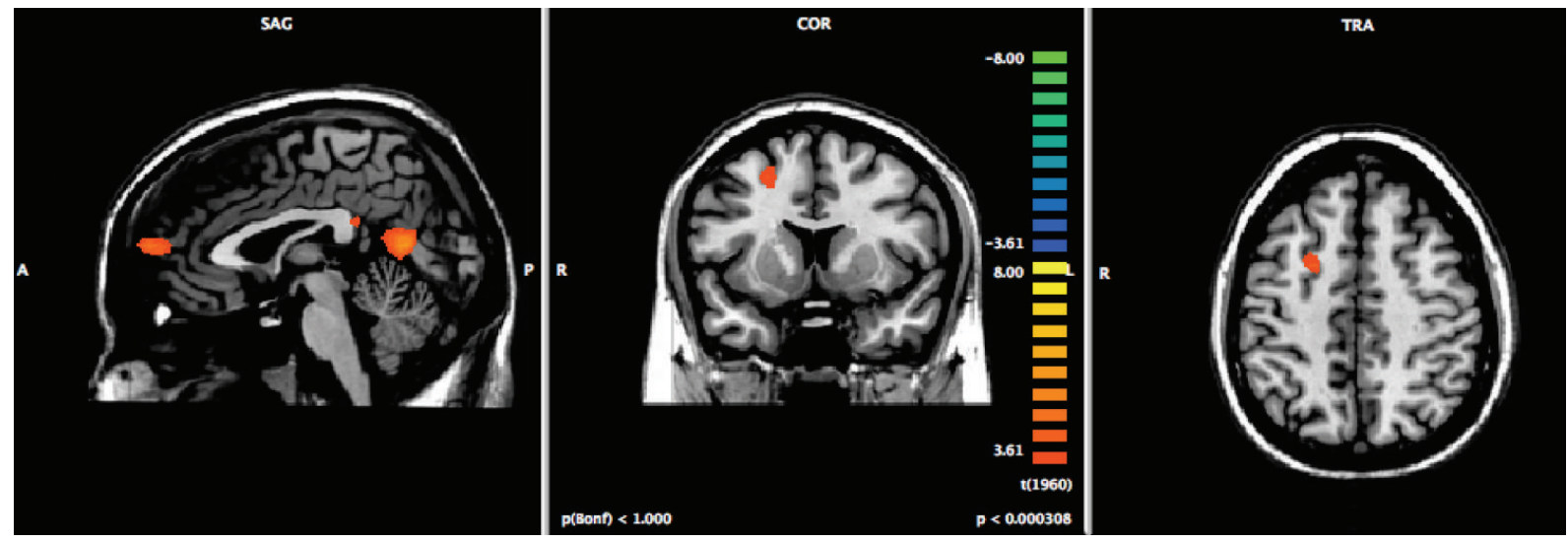

Figure 9: Pre- and posttraining differences in the motor imagery task.

TABLE 3: Talairach coordinates of activations in the motor imagery task.

\begin{tabular}{|c|c|c|c|c|c|c|c|}
\hline $\begin{array}{l}X \\
\text { coor }\end{array}$ & $\begin{array}{c} \\
\text { coor } \\
\end{array}$ & $\begin{array}{c}Z \\
\text { coor }\end{array}$ & $t$ & $P$ & & Location & \\
\hline 20.0 & 1.0 & 51.0 & 4.613 .314 & 0.000004 & Right cerebrum Frontal lobe & Subgyral & Gray matter Brodmann area 6 \\
\hline 5.0 & 61.0 & 12.0 & 6.094 .687 & 0.000000 & Right cerebrum Frontal lobe & Medial frontal gyrus & Gray matter Brodmann area 10 \\
\hline
\end{tabular}

3.4. Image Acquisition. Data acquisition was performed on a 1.5 Tesla scanner, optimized for functional imaging. Functional images were acquired using echo planar sequences, with a repetition time (TR) of $3000 \mathrm{~ms}$, an echo time (TE) of $60 \mathrm{~ms}$, and a $90^{\circ}$ flip angle. The acquisition matrix was 64 $\times 64$; the field of view (FoV) was $256 \mathrm{~mm}$. For each paradigm, a total of 100 volumes were acquired. Each volume consisted of 25 axial slices, parallel to the anterior-posterior (AC-PC) commissure line and covering the whole brain.

\section{Results and Discussion}

Imaging data were analyzed using the scanner described above.

After preprocessing, a series of steps were performed in order to allow for precise anatomical locations of brain activity to facilitate intersubject analysis. First, each subject's slice-based functional scans were coregistered with their 3D high-resolution structural scan. This process involved mathematical coregistration exploiting slice positioning stored in the headers of the raw data, as well as fine adjustments that were computed by comparing the data sets on the basis of their intensity values: if needed, manual adjustments were also performed. Second, each subject's 3D structural data set was transformed into a Talairach space, as discussed in [36]: the cerebrum was translated and rotated into the anteriorposterior commissure plane and then the borders of the cerebrum were identified. Third, using the anatomical-functional coregistration matrix and the determined Talairach reference points, each subject's functional time course was transformed into a Talairach space and the volume time course was created. For the motor paradigm, the following procedure was performed. A multisubject multistudy design matrix was specified and each defined box-car was convolved with a predefined Hemodynamic Response Function (HRF) to account for the hemodynamic delay, as discussed in [37]. A statistical analysis using the general linear model with separate study predictors was performed on the group to yield functional activation maps during the pre- and posttests separately. All voxels activated in the pretest and those activated in the posttest were combined to create a mask excluding the rest of the cerebrum and cerebellum.

This mask was used to compute the general linear model comparing posttest activations with pretest activations in the group of subjects, since the same data set was used for mask definition and subsequent statistical tests.

A comparison of imaging data obtained before and after training revealed activations in motor imagery task, that is, posttest increased hemodynamic responses, in right frontal gyrus, including supplementary motor area and right medial frontal gyrus (see Figure 9 and Table 3).

Figure 9 illustrates some selected fMRI results, showing differences before and after training with P.I.G.R.O. in the motor imagery task.

They suggest a cortical reorganization in motor areas, such as the supplementary motor area, precuneus, and cerebellum, after training which may show the effect of rehabilitation on the reorganization of somatotopic maps.

The passive stimulation (Bra.Di.P.O. in "active mode"), as expected, shows a robust sensorimotor, supplementary motor, and cerebellar activity plus some temporal and parietal clusters. The mean time course shows that the activity in this area is strongly correlated with the stimulation paradigm.

The active stimulation (Bra.Di.P.O. in "passive mode") is less affected by head motion noise and shows a less robust sensorimotor and supplementary motor activity and a cerebellar activation plus some thalamic, frontal, and cingulated clusters. The mean time span shows that the activity in this 
area is less correlated with the stimulation paradigm and with motion parameters.

The main finding was an increment of activation in motor areas. Indeed, the right medial frontal gyrus has been linked to memory retrieval and executive functions. In particular, it has been supposed to mediate attention between external stimuli and the internally maintained intention, that is, between stimulus-oriented and stimulus-independent processing, as discussed in [38].

This training required the subject to alternate attention from foot perception and position to the inputs provided by P.I.G.R.O. and vice versa. As far as the premotor areas are concerned, according to Fried et al. (1991), as discussed in [39], supplementary and presupplementary motor areas are linked with the intention and anticipation of the action, as discussed in [40].

Overall, fMRI images are clear and unaffected by the presence of the prototype in the resonance chamber. The results also allow understanding the suitability of the method here used with the presented device.

\section{Conclusions}

This research studies a locomotor and cognitive training using two mechatronics prototypes. In particular the authors investigate the circuits involved in motor imagery and motor learning at the level of brain plasticity in both healthy subjects and brain-damaged patients in the future.

The experiment was conducted on healthy subjects to assess the possibility of brain reorganization after locomotor training. Sensorimotor training is provided thanks to robotic prototypes developed at the Department of Mechanical and Aerospace Engineering, Politecnico di Torino.

Cognitive training consists of a set of motor imagery tasks. Changes in cortical organization are assessed using functional magnetic resonance imaging (fMRI), which allows the mapping of active processes within the brain, thus revealing the cerebral areas involved in a particular task.

In the future, various clinical tests on patients shocked by ictus and brain event will be carried on using P.I.G.R.O.

\section{Conflict of Interests}

The authors declare that there is no conflict of interests regarding the publication of this paper.

\section{Acknowledgments}

The work presented in this paper was financed with funding from the Compagnia di San Paolo project, "Active exoskeleton for functional gait rehabilitation of paretic patients," and with funding from the Piedmont regional administration project entitled "Validation of a method for gait rehabilitation for paretic patients using an active orthosis" (2006-2008). The authors thank Eng. Stefano Cagliero and Eng. Annalisa Rigazzi for their help in this research.

\section{References}

[1] F. C. Wang, C. H. Yu, and T. Y. Chou, "Design and implementation of robust controllers for a gait trainer," Proceedings of the Institution of Mechanical Engineers H, Journal of Engineering in Medicine, vol. 223, no. 6, pp. 687-696, 2009.

[2] D. P. Ferris, G. S. Sawicki, and M. A. Daley, "A physiologist's perspective on robotic exoskeletons for human locomotion," International Journal of Humanoid Robotics, vol. 4, no. 3, pp. 507-528, 2007.

[3] R. Gassert, E. Burdet, and K. Chinzei, "Opportunities and challenges in MR-compatible robotics," IEEE Engineering in Medicine and Biology Magazine, vol. 27, no. 3, pp. 15-22, 2008.

[4] N. V. Tsekos, A. Khanicheh, E. Christoforou, and C. Mavroidis, "Magnetic resonance - Compatible robotic and mechatronics systems for image-guided interventions and rehabilitation: a review study," Annual Review of Biomedical Engineering, vol. 9, pp. 351-387, 2007.

[5] R. Moser, R. Gassert, E. Burdet et al., "An MR compatible robot technology," in Proceedings of the IEEE International Conference on Robotics and Automation, pp. 670-675, 2003.

[6] E. Burdet, R. Gassert, G. Gowrishankar, D. Chapuis, and H. Bleuler, "fMRI compatible haptic interfaces to investigate human motor control," Experimental Robotics IX, vol. 21, pp. 2534, 2006.

[7] G. Belforte, G. Eula, S. Sirolli, and S. Appendino, "Design and testing of two mechatronics systems for robotized neurorehabilitation," in Proceedings of the 10th International Conference on Mechatronics and Precision Engineering, Bucarest, Romania, May 2011.

[8] K. Sacco, S. Appendino, E. Geda et al., "Designing a locomotor and cognitive training with robotic devices," in Proceedings of the EFRR 11th Congress of European Federation for Research in Rehabilitation, Riva del Garda, Italy, May 2011.

[9] G. Belforte, G. Eula, G. Quaglia, S. Appendino, F. Cauda, and K. Sacco, "MR compatible device for active and passive foot movements," in Proceedings of the 18th International Workshop on Robotics in Alpe-Adria-Danube Region (RAAD '09), Brasov, Romania, May 2009.

[10] G. Belforte and G. Eula, "Optimisation of a MR-Compatible mechatronic device useful for fMRI analysis," in Proceedings of the 21st International Workshop on Robotics in Alpe-AdriaDanube Region (RAAD '12), pp. 10-13, Naples, Italy, September 2012.

[11] G. Belforte and G. Eula, "Design of an active-passive device for human ankle movement during fMRI analysis," Proceedings of the Institution of Mechanical Engineers H: Journal of Engineering in Medicine January, vol. 226, 2011.

[12] G. Belforte, G. Eula, S. Appendino, and S. Sirolli, "Pneumatic interactive gait rehabilitation orthosis: design and preliminary testing," Proceedings of the Institution of Mechanical Engineers H: Journal of Engineering in Medicine, vol. 225, no. 2, pp. 158-169, 2011.

[13] K. Chinzei, R. Kikinis, and F. A. Jolesz, "MR compatibility of mechatronic devices: design criteria," in Medical Image Computing and Computer Assisted Intervention (MICCAI '99), vol. 1679 of Lecture Notes in Computer Science, pp. 1020-1031, Springer, Berlin, Germany, 1999.

[14] R. Gassert, A. Yamamoto, D. Chapuis, L. Dovat, H. Bleuler, and E. Burdet, "Actuation methods for applications in MR environments," Concepts in Magnetic Resonance B: Magnetic Resonance Engineering, vol. 29, no. 4, pp. 191-209, 2006. 
[15] H. Elhawary, Z. T. H. Tse, A. Hamed, M. Rea, B. L. Davies, and M. U. Lamperth, "The case for MR-compatible robotics: a review of the state of the art," International Journal of Medical Robotics and Computer Assisted Surgery, vol. 4, no. 2, pp. 105113, 2008.

[16] N. Yu, C. Hollnagel, A. Blickenstorfer, S. S. Kollias, and R. Riener, "Comparison of MRI-compatible mechatronic systems with hydrodynamic and pneumatic actuation," IEEE/ASME Transactions on Mechatronics, vol. 13, no. 3, pp. 268-277, 2008.

[17] H. Elhawary, A. Zivanovic, M. Rea et al., "A modular approach to MRI-compatible robotics," IEEE Engineering in Medicine and Biology Magazine, vol. 27, no. 3, pp. 35-41, 2008.

[18] G. S. Fischer, A. Krieger, I. Iordachita, C. Csoma, L. L. Whitcomb, and G. Fichtinger, "MRI compatibility of robot actuation techniques-a comparative study," in Medical Image Computing and Computer-Assisted Intervention (MICCAI '08), vol. 5242 of Lecture Notes in Computer Science, no. 2, pp. 509-517, Springer, Berlin, Germany, 2008.

[19] C. Wienbruch, V. Candia, J. Svensson, R. Kleiser, and S. S. Kollias, "A portable and low-cost fMRI compatible pneumatic system for the investigation of the somatosensensory system in clinical and research environments," Neuroscience Letters, vol. 398, no. 3, pp. 183-188, 2006.

[20] N. Yu, W. Murr, A. Blickenstorfer, S. Kollias, and R. Riener, "An fMRI compatible haptic interface with pneumatic actuation," in Proceedings of the IEEE 10th International Conference on Rehabilitation Robotics (ICORR '07), pp. 714-720, Noordwijk, The Netherlands, June 2007.

[21] C. Raoufi, A. A. Goldenberg, and W. Kucharczyk, "A new hydraulically/pneumatically actuated mrcompatible robot for MRI-guided neurosurgery," in Proceedings of the 2nd International Conference on Bioinformatics and Biomedical Engineering (ICBBE '08), pp. 2232-2235, Shanghai, China, May 2006.

[22] B. J. MacIntosh, R. Mraz, N. Baker, F. Tam, W. R. Staines, and S. J. Graham, "Optimizing the experimental design for ankle dorsiflexion fMRI," NeuroImage, vol. 22, no. 4, pp. 1619-1627, 2004.

[23] S. Francis, X. Lin, S. Aboushoushah et al., "fMRI analysis of active, passive and electrically stimulated ankle dorsiflexion," NeuroImage, vol. 44, no. 2, pp. 469-479, 2009.

[24] ISO, 7250-1: Basic human body measurements for technological design. Part 1: Body measurement definitions and landmarks.

[25] ISO/TR, 7250-2: Basic human body measurements for technological design. Part 2: Statistical summaries of body measurements from individual ISO populations.

[26] K. Kubo, T. Miyoshi, A. Kanai, and K. Terashima, "Gait rehabilitation device in central nervous system disease: a review," Journal of Robotics, vol. 2011, Article ID 348207, 14 pages, 2011.

[27] I. Díaz, G. G. Gil, and E. Sánchez, "Lower-limb robotic rehabilitation: literature review and challenges," Journal of Robotics, vol. 2011, Article ID 759764, 11 pages, 2011.

[28] G. S. Sawicki, K. E. Gordon, and D. P. Ferris, "Powered lower limb orthoses: Applications in motor adaptation and rehabilitation," in 2Proceedings of the IEEE 9th International Conference on Rehabilitation Robotics (ICORR '05), pp. 206-211, Chicago, Ill, USA, July 2005.

[29] P. Beyl, M. van Damme, R. van Ham, and D. Lefeber, "Design and control concepts of an exoskeleton for gait rehabilitation," in Proceedings of the 2nd Biennial IEEE/RAS-EMBS International Conference on Biomedical Robotics and Biomechatronics (BioRob '08), pp. 103-108, Scottsdale, Ariz, USA, October 2008.
[30] D. Surdilovic, J. Zhang, and R. Bernhardt, "STRING-MAN: wire-robot technology for safe, flexible and human-friendly gait rehabilitation," in Proceedings of the IEEE 10th International Conference on Rehabilitation Robotics (ICORR '07), pp. 446453, Noordwijk, The Netherlands, June 2007.

[31] X. Zhang, C. Yang, J. Zhang, and Y. Chen, "A novel DGO based on pneumatic exoskeleton leg for locomotor training of paraplegic patients," in Intelligent Robotics and Applications, Lecture Notes in Computer Science, pp. 528-537, Springer, Berlin, Germany, 2008.

[32] G. Belforte, G. Eula, S. Appendino, G. C. Geminiani, and M. Zettin, "Tutore attivo per neuroriabilitazione motoria degli arti inferiori, sistema comprendente tale tutore e procedimento per il funzionamento di tale sistema," Patent TO2012A000226, 2012.

[33] J. Perry, Gait Analysis-Normal and Pathological Function, SLACK Incorporated, 1992.

[34] S. Ionta, A. Ferretti, A. Merla, A. Tartaro, and G. L. Romani, "Step-by-step: the effects of physical practice on the neural correlates of locomotion imagery revealed by fMRI," Human Brain Mapping, vol. 31, no. 5, pp. 694-702, 2010.

[35] F. Malouin and C. L. Richards, "Mental practice for relearning locomotor skills," Physical Therapy, vol. 90, no. 2, pp. 240-251, 2010.

[36] J. Talairach and P. Tournoux, Co-Planar Stereotaxic Atlas of the Human Brain: 3-Dimensional Proportional System: An Approach to Cerebral Imaging, Thieme, Stuttgart, Germany, 1988.

[37] G. M. Boynton, S. A. Engel, G. H. Glover, and D. J. Heeger, "Linear systems analysis of functional magnetic resonance imaging in human V1," Journal of Neuroscience, vol. 16, no. 13, pp. 4207-4221, 1996.

[38] O. Baumann and M. W. Greenlee, "Effects of attention to auditory motion on cortical activations during smooth pursuit eye tracking," PLoS ONE, vol. 4, no. 9, Article ID e7110, 2009.

[39] I. Fried, A. Katz, G. McCarthy et al., "Functional organization of human supplementary motor cortex studies by electrical stimulation," Journal of Neuroscience, vol. 11, no. 11, pp. 36563666, 1991.

[40] K. Sacco, F. Cauda, S. Duca et al., "A combined robotic and cognitive training for locomotor rehabilitation: evidences of cerebral functional reorganization in two chronic traumatic brain injured patients," Frontiers in Human Neuroscience, pp. 19, 2011. 

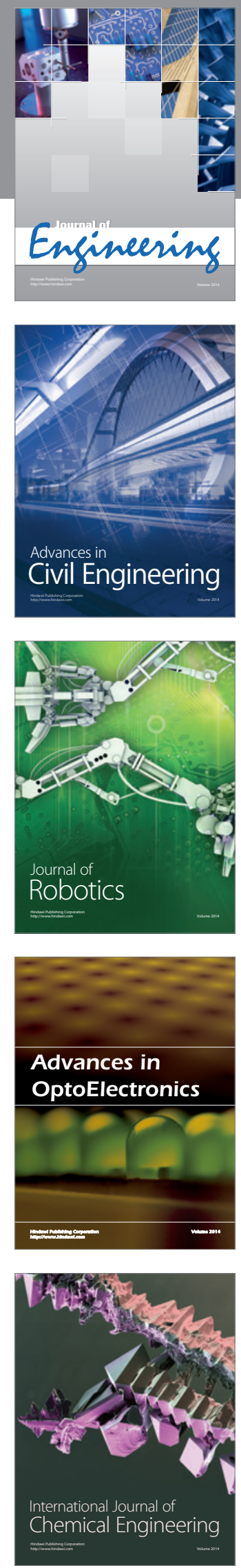

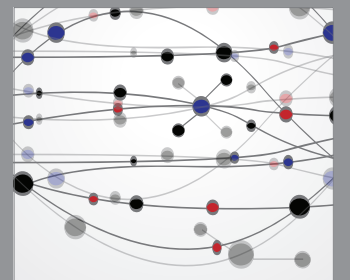

The Scientific World Journal
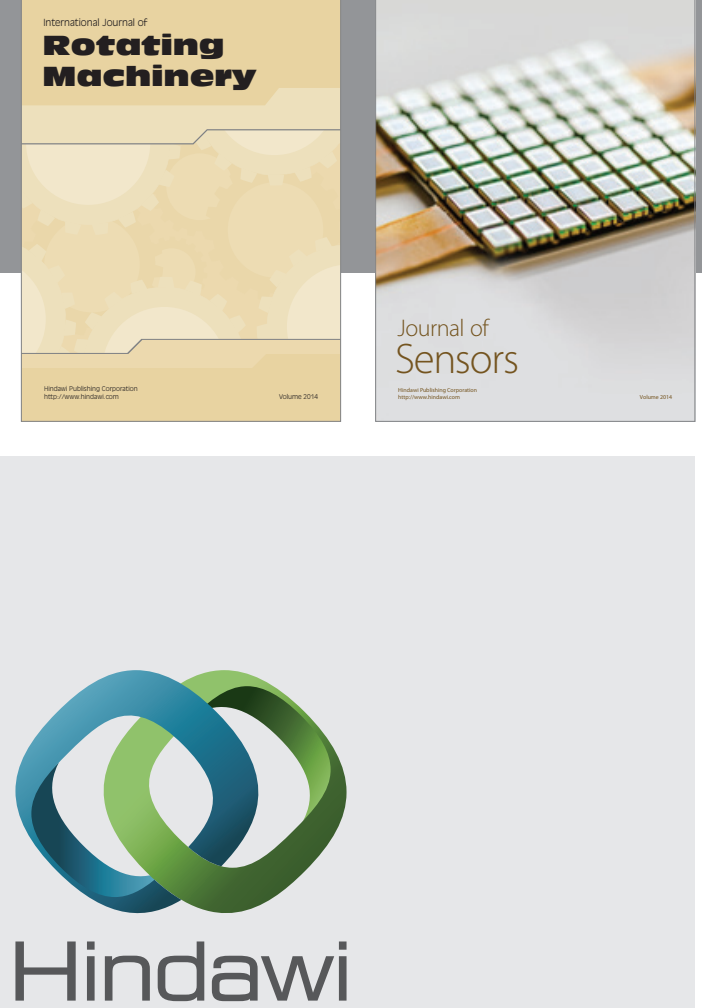

Submit your manuscripts at http://www.hindawi.com
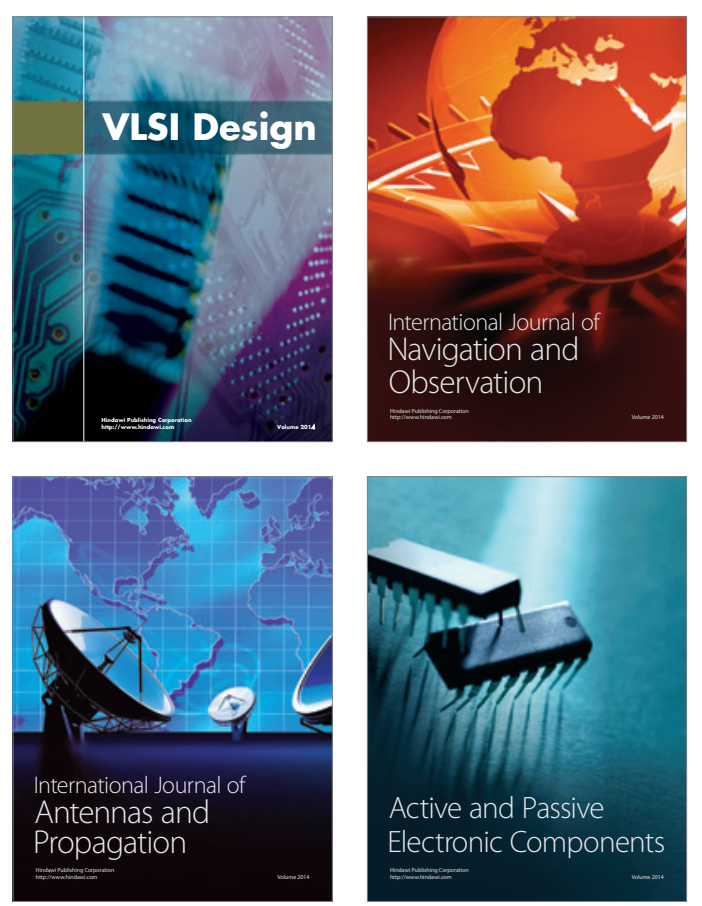
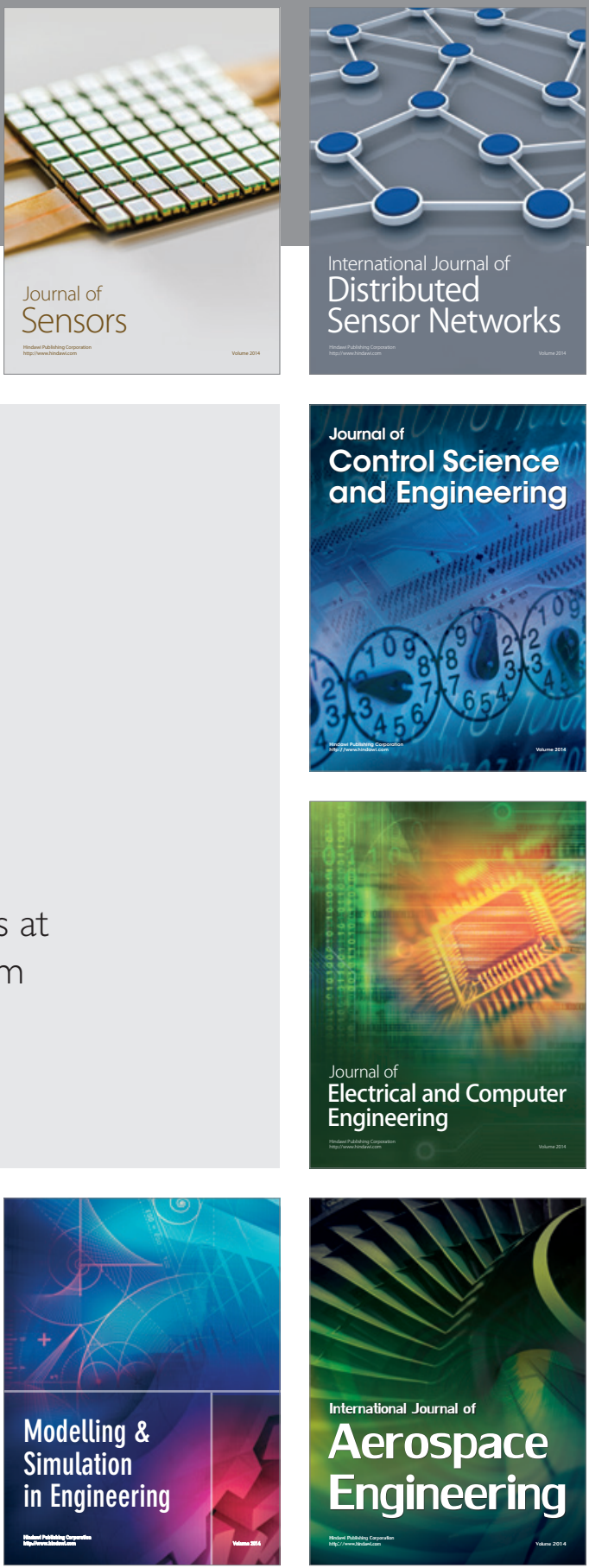

Journal of

Control Science

and Engineering
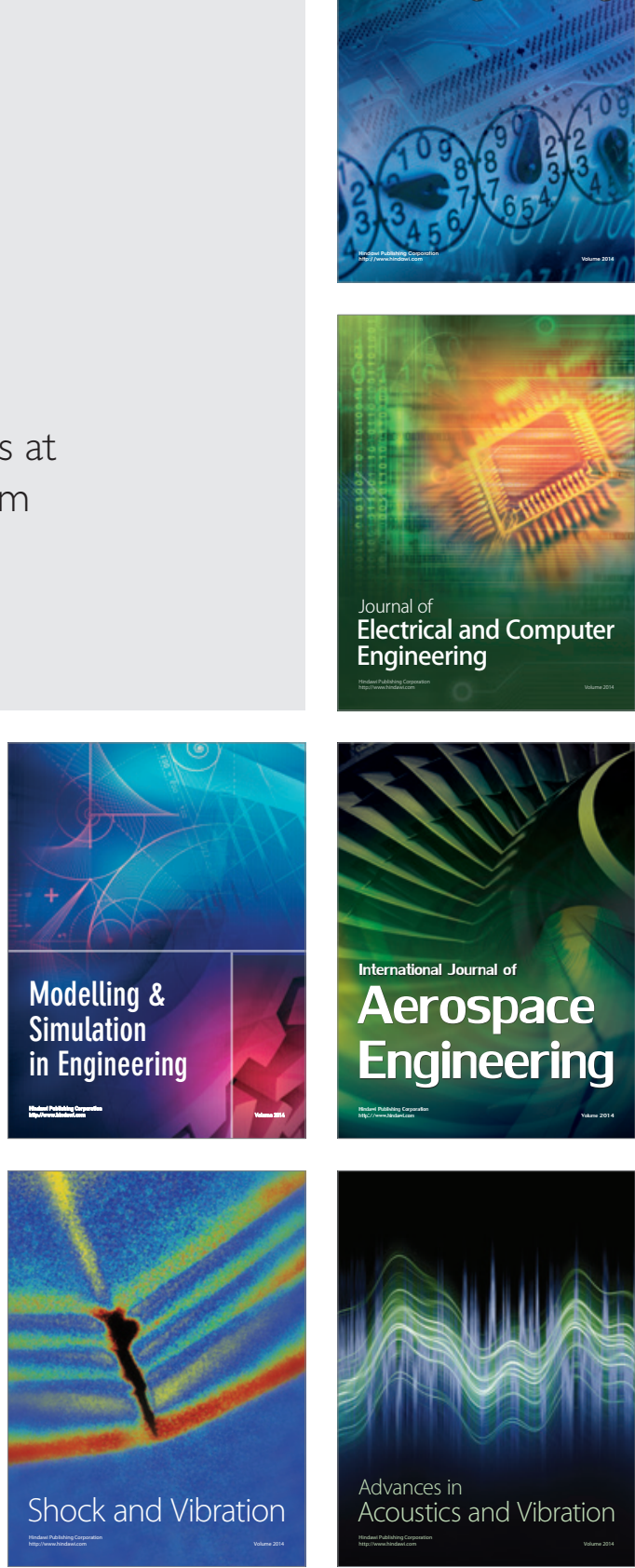